\title{
Human Pluripotent Stem Cells: Advances in Chondrogenic Differentiation and Articular Cartilage Regeneration
}

\author{
Rosa M. Guzzo ${ }^{1}$ Michael B. O’Sullivan ${ }^{1}$
}

Published online: 7 July 2016

(C) Springer International Publishing AG 2016

\begin{abstract}
Articular chondral lesions are major risk factors for the development of osteoarthritis (OA). Multiple adult cellbased approaches have been attempted to restore hyaline cartilage and prevent progressive degeneration; however, the formation of permanent cartilage has not yet been achieved. A scalable source of cartilage progenitors may have far-reaching potential to advance joint cartilage therapy as well as disease modeling and would be expected to facilitate the discovery of novel therapeutics to stimulate cartilage regeneration or prevent degeneration. Because of their unlimited proliferative capacity and pluripotency, human pluripotent stem cells have become an attractive therapeutic option as a source for consistently uniform cells with high chondrogenic capacity. This review focuses on the recent progress using developmentbased paradigms to control the differentiation of human pluripotent stem cells to an articular chondrocyte fate. We highlight recent findings that demonstrate the promise for using pluripotent stem cell-based replacement for hyaline cartilage repair.
\end{abstract}

Keywords Articular cartilage repair - Embryonic stem cells . Induced pluripotent stem cells · Mesenchymal stem cells . Osteoarthritis $\cdot$ Regeneration

This article is part of the Topical Collection on Stem/Progenitor Cells Biology and Regeneration

Rosa M. Guzzo

guzzo@uchc.edu

Michael B. O’Sullivan

osullivan@uchc.edu

1 Department of Orthopaedic Surgery, UConn Health Center, 263 Farmington Avenue, Farmington, CT, USA

$\begin{array}{ll}\text { Abbreviations } \\ \text { ACAN } & \text { Aggrecan } \\ \text { ACI } & \text { Autologous chondrocyte implantation } \\ \text { bFGF } & \text { Basic fibroblast growth factor } \\ \text { BMP } & \text { Bone morphogenetic protein } \\ \text { BMPR1B } & \text { Bone morphogenetic protein receptor 1 beta } \\ \text { BMSC } & \text { Bone marrow-derived MSCs } \\ \text { ESC } & \text { Embryonic stem cells } \\ \text { GDF } & \text { Growth and differentiation factor } \\ \text { iPSC } & \text { Induced pluripotent stem cells } \\ \text { ISCT } & \text { International Society for Cellular Therapy } \\ \text { LIF } & \text { Leukemia inhibitory factor } \\ \text { MSCs } & \text { Mesenchymal stem cells } \\ \text { OA } & \text { Osteoarthritis } \\ \text { PDGF } & \text { Platelet-derived growth factor } \\ \text { PSC } & \text { Pluripotent stem cells } \\ \text { TGF } & \text { Transforming growth factor } \\ \text { VEGF } & \text { Vascular endothelial growth factor }\end{array}$

\section{Introduction}

Osteoarthritis (OA) is a common and debilitating joint disease for which primary risk factors are traumatic joint injury or mechanical disruption of joint tissues. There is currently no cure for OA. Although the true prevalence of articular chondral lesions in the general public is unknown, approximately $60 \%$ of patients undergoing knee arthroscopy have evidence of cartilage lesions $[1,2]$. The natural history of articular cartilage lesions is poorly understood, as the means for evaluating these lesions (MRI, arthroscopy) are not commonly used for surveillance due to cost implications. A prevailing notion is that chondral lesions increase in size and predispose patients to developing OA. Supporting this idea is evidence that articular cartilage has limited regenerative 
capacity, is poorly vascularized, and has a small cell-to-matrix volume and a very low mitotic rate [3]. Treatment options for OA patients such as total joint arthroplasty provide excellent outcomes by ameliorating pain and improving function. However, many patients sustain cartilage injuries when they are young and are not good candidates for these procedures as they would outlive their implant and would require multiple revision surgeries [2, 4-6]. Currently, the prevalence of patients living in the USA with a total hip or total knee arthroplasty is estimated to be 7.2 million, which is higher than the prevalence of stroke ( 6.8 million) and heart failure (5.1 million) and approaches that of myocardial infarction (7.6 million) [7]. Thus, OA is an important public health issue, and there remains an urgent and growing need to develop regenerative techniques for articular cartilage to treat symptomatic patients and potentially circumvent the onset of OA among individuals predisposed to developing OA.

Multiple strategies have been attempted for joint cartilage surface restoration, with the goal of improving joint function and delaying or preventing degeneration. However, regeneration of hyaline cartilage has not been achieved. Introduced by Brittberg et al. in 1994, implantation of in vitro expanded autologous human articular chondrocytes (hACs) is a widely used surgical procedure to treat focal chondral lesions in the knee joint [8]. Autologous chondrocyte implantation (ACI) involves extraction of a small cartilage biopsy from a nonload-bearing site within the affected knee joint and in vitro expansion of the isolated chondrocytes using "good manufacturing practice" (GMP) laboratory procedures, which is then followed by implantation of these cells to the defect site in a second surgical procedure [8]. The repair tissue is often fibrocartilaginous, with little hyaline cartilage restoration $[9$, 10]. Fibrocartilage generally deteriorates over time because of its inferior structural and mechanical properties, which increases the likelihood of OA and the need for further surgical intervention.

Adult mesenchymal stem cells, commonly isolated from bone marrow, have been extensively examined as an alternative to either autologous or allogeneic chondrocytes for regeneration of articular cartilage. These cells offer advantages of ease of harvest using minimally invasive procedures, low immunogenicity, high proliferative indices, and an intrinsic chondrogenic capacity that can be exploited to yield vast quantities of chondroprogenitors to repair cartilage defects [11]. However, chondrogenic differentiation in bone marrow-derived MSCs (BMSCs) follows an endochondral pathway, yielding transient cartilage expressing markers of hypertrophy, mineralization, and catabolic enzymes detrimental to maintenance of permanent cartilage [12-16]. Donor-todonor variability, as well as age-dependent decline in replicative and differentiation capacity, may also contribute to variable clinical outcomes when using adult mesenchymal stem cell (MSC) for cartilage repair $[17,18]$. Over the past decade, accumulating evidence also supports the presence of a resident stem/progenitor cell population within intact articular cartilage with the potential to respond to injury and to repair small lesions [19-26]. However, the means through which the expansion and differentiation of these cells can be effectively controlled to promote endogenous tissue repair remains unknown.

\section{The Induced Pluripotent Stem Cell Revolution}

Embryonic stem cells (ESCs) provide the unprecedented means to study mechanisms of human lineage commitment and cell specification; however, obvious ethical issues and a lack of patient specificity impede their clinical utility. Yamanaka's Nobel prize winning discovery that pluripotency can be induced in virtually any somatic cell through the transient ectopic expression of four reprogramming factors (Oct3/4, Klf4, Sox2, Myc; Nanog and Lin28 can replace cMyc and Klf4) [27-29] has transformative potential for personalized regenerative medicine and disease modeling. These induced pluripotent stem cells (iPSCs) offer an ethically unencumbered source of patient-specific cells, which display hallmark features of ESCs, including expression of canonical pluripotency markers (OCT4, SSEA-4, NANOG, TRA-1-60, TRA-I-80). iPSCs also provide unlimited self-renewal and the ability to form derivatives of each of the three germ layers. Viral transduction has been the approach traditionally used to introduce the reprogramming factors, though this earlier approach may hinder their clinical use in patients. However, recent advances in reprogramming now offer safe and efficient transgene-free reprogramming techniques using synthetic messenger RNA, microRNA, proteins, and supplementation with small molecule compounds [30-33].

Unlike human primary cells, specialized cell types can be generated from highly renewable sources of iPSCs. Thus, cell reprogramming provides the unique ability to model diverse human diseases and to perform large-scale small molecule and drug screening using cells from panels of human patients representing the spectrum of a particular disease and drug response [34]. For instance, a drug screen using a library of human iPSC-derived cardiomyocytes indicated that healthy and diseased individuals exhibit different susceptibilities to cardiotoxic drugs [35]. Such studies have tremendous value as human disease-specific iPSC-derived cells may provide more accurate prediction of adverse drug responses as compared to standard clinical assays. Moreover, animal models often lack human disease relevance; thus, the use of human iPSCs may better capture the complexity of disease pathophysiology [34]. Applications of iPSCs and ESCs in highthroughput small molecule screening have also led to the identification of novel molecules that promote differentiation to specific lineages, including cardiomyocytes and insulin- 
producing pancreatic b cells [36-38], as well as the identification of molecules that inhibit pluripotency [39]. These discoveries are critical for developing effective cell replacement strategies, as well as for the selective removal of undifferentiated cells prior to in vivo transplantation of iPSC-derived specialized cell types. Indeed, a major step toward the realizing the regenerative potential of iPSCs came in 2014 with a pioneering clinical study in Japan, where a patient suffering from age-related macular degeneration received the first iPSC-derived transplant. Progress toward the use of human iPSCs in (i) cartilage disease modeling, (ii) the discovery of new drugs that promote cartilage formation or prevent cartilage degeneration, and (iii) cartilage regenerative therapy is predicated upon the derivation of homogeneous articular-like chondrocytes which exhibit functional and genomic integrity. Knowledge gleaned from the developmental biology of articular cartilage has been applied to establish the molecular signaling and culture conditions to promote differentiation of human ESCs and iPSCs toward the chondrogenic lineage $[40 \bullet, 41 \bullet \bullet, 42 \bullet, 43-50]$. Indeed, human iPSCs may provide an unparalleled source of progenitors to model articular development, since the bona fide cartilage progenitors present only early in development are otherwise inaccessible [40 $]$. Below, we highlight recent findings that demonstrate the promise for using pluripotent stem cell (PSC)-based replacement for hyaline cartilage repair.

\section{Development-Based Strategies to Recapitulate Chondrogenesis in Human Pluripotent Stem Cells}

Chondrogenesis during embryogenesis is a tightly regulated process involving the recruitment and migration of mesenchymal cells, condensation of progenitors to form compact nodules, commitment of the primordial mesenchymal cells to the chondrocyte lineage, and differentiation to proliferating prechondrocytes [51, 52]. Growth factors are critical for regulating the discrete stages of the chondrogenic differentiation program. For instance, fibroblast growth factor (FGF2) signaling plays a key role in the development of mesenchymal progenitors, while BMP4 has been shown to mediate mesoderm specification and instruct the uncommitted progenitors to the chondrogenic lineage [53]. Following commitment of chondrogenesis, the cells undergo differentiation via the endochondral ossification pathway that leads to the formation of bone or are differentiated toward the articular cartilage fate. Based on cell lineage tracking experiments in developmental model systems, articular chondrocytes, as well as other components of the synovial joint, are known to arise from a specialized population of condensed mesenchymal progenitors known as interzone cells [54]. Signaling through the transforming growth factor (TGF) $\beta$ pathway is required for the initial formation of joint interzone cells and the maintenance of phenotypic interzone markers such as Noggin, Wnt9a, and growth and differentiation factor-5 (Gdf5) [55]. Downstream intracellular signaling from BMPs and TGF $\beta$ exerts their chondrogenic effects on progenitor cells through distinct gene regulatory pathways, such as regulation of SOX and RUNX family of transcription factors. Articular chondrocytes do not undergo hypertrophy and maturation, as seen in growth plate chondrocytes. It is well established that TGF $\beta$ inhibits chondrocyte maturation, whereas BMP signaling exerts positive effects on chondrocyte maturation. Thus, the sequential or concurrent activation of TGF $\beta$ and BMP pathways has been used as a strategy to promote chondrogenesis in various adult and pluripotent stem cells.

Several studies have identified the molecular signature of the primitive human mesodermal progenitor populations from human PSCs, and this knowledge has facilitated the development of more efficient strategies to promote chondrogenic differentiation. Earlier studies by Evseenko and colleagues identified the $\mathrm{CD} 326^{-} \mathrm{CD} 56^{+}$population as the earliest multipotent mesoderm-committed progenitor population that arises from day 3.5 differentiated human ESCs during the process of epithelial-to-mesenchymal transition mediated by recombinant Activin A, BMP4, VEGF, and FGF2 [56]. In vitro functional assays demonstrated the full potential of this population, giving rise to all mesodermal lineages including chondrocytes. Additional studies have revealed that precise stage-specific modulation of multiple signaling pathways downstream of the early mesendoderm population can recapitulate the human developmental chondrogenic program in ESCs and iPSCs $[40 \bullet, 41 \bullet \bullet, 42 \bullet, 46,57 \bullet \bullet, 58]$. Oldershaw et al. devised a three-step, chemically defined and serum-free protocol for directed differentiation of human ESCs through a transient primitive streak/mesendoderm stage, followed by controlled differentiation to a multipotent mesoderm, and subsequent differentiation of the mesoderm intermediates to chondrocytic cells within three-dimensional aggregates [42•]. Requiring temporal supplementation of seven different growth factors (Activin A, bFGF, WNT3a, BMP4, NEUROTROPHIN-4 (NT4), FOLLISTATIN, growth and differentiation factor-5 (GDF)), this approach has been used by multiple groups for efficient production of differentiated chondrocytes from independent lines of human embryonic stem cells (hESCs) and iPSCs $[40 \bullet, 41 \bullet \bullet, 42 \bullet, 46,57 \bullet \bullet, 58]$. Lee et al. further optimized this approach to achieve improved survival and chondrogenic differentiation of human iPSCs, which showed enrichment of SOX ${ }^{+} \mathrm{CD} 140^{+} \mathrm{CD} 44^{+}$chondrocytes expressing phenotypic markers of articular chondrocytes (i.e., WNT9a, SOTDC1, $C O L 2 A 1, A C A N)$, while lacking expression of markers of hypertrophic chondrocytes $(C O L 10 A 1)$ and fibrocartilage (COL1A1) [58]. Transplantation of these iPSC-derived chondrocytes into immunodeficient mice was found to maintain a stable cartilage phenotype without evidence of tumorigenicity after 4 weeks [58]. A comprehensive transcriptional 
profiling of the intermediate populations during differentiation toward chondrocyte maturation provided new mechanistic insights into how the SOX9 regulatory network operates in the early mesodermal and mature chondrocyte fates [58]. Using iPSC lines generated by episomal reprogramming, Yamashita et al. devised a similar but simplified approach based on the initial mesendodermal differentiation in response to WNT3a and Activin A [42•] but followed this with differentiation to the chondrocytic lineage by temporal exposure of suspension cultures to ascorbic acid, BMP2, TGF $\beta 1$, and GDF5 [59••]. The use of a chondrocyte-specific COL11a2-eGFP reporter and gene expression analyses confirmed that this approach generated a highly purified population of lubricin/PRG4 expressing articular-like chondrocytes, which lacked the expression of type X collagen [59••].

In 2013, Evessenko and colleagues were the first to characterize the earliest stages of human chondrogenic development from human embryonic distal limbs, and the developing embryonic femoral bone epiphyses, by applying microarray screening and immunophenotypic profiling of laser-captured cells [40॰]. Comprehensive transcriptional profiling and functional assays led to the identification of unique combinations of cell surface markers that can identify and differentiate between the earliest prechondrocytes $\left(\mathrm{CD} 146^{\text {low/neg }} \mathrm{CD} 166^{\text {low } /}\right.$ $\left.{ }^{\text {neg }} \mathrm{CD} 73^{+} \mathrm{CD} 44^{\text {low }}\right)$, immature periarticular chondrocytes $\left(\mathrm{BMPR} 1 \mathrm{~B}^{+} \mathrm{LIFR}^{+}\right.$), and hypertrophic chondrocytes (BMPR1B ${ }^{\text {neg }}$ LIFR $^{\text {neg }}$ ). Based on these results, it was then possible to isolate primitive prechondrocytes and definitive resting chondrocytes from differentiating PSC cultures. Moreover, this study revealed that temporal modulation of LIF, TGF $\beta$, and BMP signaling pathways in iPSC-derived cartilage progenitors could promote the development of immature chondrocytes, while concurrently inhibiting chondrocyte hypertrophy and maturation. Subsequent work by the Keller Laboratory has also provided critical insights into the molecular landmarks and requisite signaling for controlled specification of human ESCs to the articular chondrocyte versus hypertrophic chondrocyte fate $[41 \bullet \bullet$. Sustained TGF $\beta 3$ signaling in human ESC-derived mesenchymal progenitors in micromass was needed to specify a population expressing hallmark markers of interzone cells (i.e., GDF5, WNT9A, $E R G, S O S T D C 1, D C X)$ and subsequently of articular chondrocytes (i.e., $P R G 4 /$ lubricin, cartilage intermediate layer protein $2 / C I L P 2, C O L 2 A 1)$ [41 •*]. In contrast, treatment with BMP4 promoted a hypertrophic chondrocyte fate, confirmed by high level of expression of hypertrophy genes including alkaline phosphatase (ALP), COL10A1, and RUNX2, as well as increased apoptosis indicative of an endochondral pathway. Moreover, distinct differences in the quality of cartilage formed by the TGF $\beta$ - versus BMP4-treated cells were observed following subcutaneous implantation of the two populations in immune-deficient mice. Consistent with their in vitro observations, 12-week grafts from BMP4-treated cells were found to initiate endochondral ossification, whereas TGF $\beta 3$-treated grafts maintained a proteoglycan- and type II collagen-rich ECM with expression of lubricin/PRG4, with no evidence of chondrocyte hypertrophy or calcification. Thus, there is a continued promise that this approach may be translated for generation of transplantable cells to regenerate damaged hyaline cartilage.

\section{Human Pluripotent Stem Cells Are a Source of Multipotent Mesenchymal-Like Progenitors}

Methods for specification and prospective isolation of cartilage-committed progenitors from a heterogeneous population of differentiating human PSCs are technically challenging, as they require temporal fine-tuning of multiple signaling pathways. Thus, alternative approaches for generating scalable populations of multipotent cells with high chondrogenic capacity have also been explored. Applying diverse methodologies, multiple groups have now shown that the epithelialto-mesenchymal transition step in differentiation of human iPSCs and hESCs could be achieved by (a) differentiating with or without an EB intermediate (EB) [43, 44, 60-65], (b) enriching an intermediate hemangioblast population [66, 67], (c) adding a small molecule inhibitor of TGF signaling [68], and (d) co-culturing with mesenchymal cell lines [69]. Collectively, these approaches have demonstrated that the loss of pluripotency and acquisition of the characteristic MSC-like morphology is associated with expression of MSC antigens (positive for CD90, CD73, CD105, CD29, CD44, CD49, CD166 and negative for CD34 and CD45), as well as the capacity for multi-lineage differentiation in vitro and in vivo. Chondrogenic differentiation within 3D cultures of PSCderived MSCs has been induced via singular and combined treatment with growth factors, known to play important roles in initiating embryonic mesenchymal condensations and cartilage nodule formation (i.e., TGF $\beta 1$, TGF $\beta 3$, BMP2, BMP4, BMP6, and GDF5) [44, 46, 70-72]. However, few studies have demonstrated controlled differentiation of human PSCderived MSCs to articular-like chondrocytes for cartilage defect repair [61].

A key question in the field is whether there exists molecular and functional equivalency between human iPSC-derived MSCs and adult MSCs. To gain insight, Diederich et al. performed side-by-side genomic and functional comparisons of adult BMSCs and MSCs generated from human BMSCiPSCs. The study design used human donor-specific iPSCs generated from BMSCs, as well as multiple methods to derive MSCs from iPSCs to circumvent confounding effects attributed to donor variability or method of MSC derivation. Comparative analyses revealed distinct transcriptomic as well as functional differences between the two sources [73]. iPSCderived MSCs were generally less responsive to traditional 
BMSC chondrogenic differentiation protocols [73]. Although functional outcomes in vivo were not available, the authors speculated that compliance with criteria put forth by the International Society for Cellular Therapy (ISCT) [74] may not be sufficient to confirm derivation of bonafide MSCs from iPSCs. In another comparative in vitro study using combinations of TGF $\beta 3$, BMP, and PDGF to drive chondrogenic differentiation, superior formation of hyaline-like cartilage tissue was observed in human ESC-derived paraxial mesoderm-like cells as compared to either adult MSCs or hESC-derived MSC-like cells (the latter lacking mesendoderm identity) [45]. These functional disparities in vitro may point to a need for more sophisticated factor-based regimens to direct lineagespecific hPSC differentiation or the need for alternative methods to enrich the chondrogenic progenitors from hPSCs $[45,73]$.

Others have postulated that human iPSCs bear an epigenetic advantage over adult stem cells for large-scale generation of chondrocytes that do not exhibit a propensity for hypertrophy. While examining differences in chondrogenesis between BMSC and human induced pluripotent cells (hiPSCs), Ko and colleagues demonstrated greater expression of signature chondrogenic markers (i.e., SOX9, COL2A1, ACAN) and lower levels of hypertrophic markers (i.e., COL10A1, RUNX2) in chondro-induced iPSCs versus BMSC pellets [64]. Differences in the maturation status were attributed to epigenetic disparities between the two cell sources at the level of DNA methylation. Enhanced methylation of the COL10A1 gene promoter was also present in human iPSC-derived chondrocytes compared with BMSCs, which corresponded to reduced COL10A1 gene expression seen in human iPSCderived chondrocytes [64]. Whether the epigenetic disparities among the cell sources may be used to predict a superior regenerative potential or better quality cartilage in vivo remains to be determined.

\section{Epigenetic Regulation of Chondrogenic Differentiation}

Epigenetic mechanisms including DNA methylation, chromatin remodeling, and histone modifications serve a fundamental role in the control of stem cell maintenance and differentiation. The epigenomic landscape in human PSCs, their differentiated progeny, and a broad range of adult cell types including BMSC-derived chondrocytes have been systematically determined, revealing developmental and tissue-specific regulation of lineage-control genes. It is anticipated that targeting the epigenetic machinery may present new avenues to finetune chondrocyte cell fate decisions in differentiating PSCS. Histone posttranslational modifications (PTM) play a fundamental role in the control of gene expression by altering chromatin conformation and accessibility of the transcriptional machinery $[75,76]$. Combinations of PTMs such as acetylation $(\mathrm{ac})$ and methylation $(\mathrm{me})$ on lysine $(\mathrm{K})$ residues in histone $\mathrm{H} 3$ constitute an "epigenetic code" for transcriptional activation or repression. Genome-wide mapping of the quantified epigenetic changes during chondrogenic differentiation in adult bone marrow-derived MSCs revealed that histone PTMs constitute a primary mechanism for induction of cartilage lineage genes in differentiating mesenchymal progenitors $[77 \bullet, 78]$. Several recent studies have also indicated that targeting of chemically modifiable chromatin modifiers, such as histone lysine demethylases (i.e., KDM4B, JMJD3, PHF2), may provide new strategies to improve chondrogenesis and control maintenance of the chondrocyte phenotype [79-83]. However, the epigenetic regulation of chondrogenesis in human PSCs remains largely unexplored. Thus, with emerging knowledge of the epigenetic landmarks and critical modifiers distinguishing the distinct stages of chondrogenic lineage progression, we may be better poised to limit the risk of tumorigenicity, enhance chondrogenic capacity, and exert more precise control over the specification of articular-like chondrocytes in human pluripotent stem cells.

\section{Pluripotent Stem Cell-Mediated Repair of Articular Cartilage Damage}

Scaffold-cell complexes and scaffoldless approaches have been used to assess the potential of human pluripotent stem cells in engineered cartilage formation and cartilage defect repair [41 •, 57••, 58, 59••, 61, 64, 84-86, 87•, 88]. Several recent studies demonstrated the efficacy of human ESCs and iPSCs to mediate the repair of cartilage defects in various animal models. Fibrin gelencapsulated hESC-derived chondroprogenitors generated via a chemically defined and feeder-free culture system promoted the repair of focal defects in rats with hyaline-like cartilage, without evidence of tumor formation [85]. However, hESC-mediated cartilage repair was not equivalent to uninjured cartilage. TGF $\beta 3$ mediated chondro-induced iPSCs implanted as pellets or within alginate hydrogel promoted the restoration of the articular cartilage surface 12 weeks following implantation into osteochondral defects in immunosuppressed rats [64]. In other studies, histological analyses also indicated reduced proteoglycan content in the iPSC-derived tissue when compared with adjacent host tissue [64]. Yamashita et al. recently used scaffold-free, stagedependent chondrocyte particles derived from COL11A2-EGFP iPSCs to evaluate their ability to repair osteochondral defects in nude rats and minipigs [59•*]. Cartilaginous particles differentiated from iPSC-derived mesendodermal cells treated with TGF $\beta$, GDF5, and BMP2 were transplanted into defects created in the articular cartilage of SCID rats, without tumor formation or ectopic tissue formation. Immunohistochemical showed strong expression of type II collagen at 12 weeks post-implantation, as well as preservation of hyaline-like cartilage structure, and good 
integration between tissue formed by transplanted cells and rat articular cartilage. The hiPSC-derived chondrocytes also showed promise in filling defects in minipigs 4 weeks after implantation, yet demonstration of long-lasting mechanically stable hyaline-like cartilage using this approach will require further studies.

Although hyaline-like cartilage regeneration in vivo has been demonstrated following orthotopic transplantation of pluripotent stem cell-derived chondroprogenitor and chondrocytes, without signs of tumor growth $[57 \bullet \bullet, 59 \bullet$, 64], the potential carry-over of undifferentiated and undefined cell types remains a valid concern. Saito and colleagues recently reported development of a large immature teratoma 16 weeks following transplantation of iPSC-derived chondrocytes into a full-thickness cartilage defect in the murine knee joint [87॰]. This corresponded to an incidence of $6.7 \%$. These findings emphasize the critical need for refined and standardized methods to purify homogenous populations of human pluripotent stem cell-derived chondrocytes, as well as more thorough characterizations of the transplants over an extended follow-up period, and removal of undifferentiated cells prior to clinical use.

Transdifferentiation of adult cells has been explored as an alternative approach to generate large quantities of chondrogenic progenitors that do not carry risk of teratoma formation [89•]. It is currently thought that transdifferentiation, or lineage conversion, can occur without transition through a pluripotent state. This possibility would provide an additional level of safety; however, this process has been called into question by several recent studies [90, 91]. Outani et al. determined that retroviral delivery of two reprogramming factors (c-MYC, KLF4) and the master cartilage regulatory SOX9 was sufficient for lineage reprogramming of human dermal fibroblasts into proliferative induced chondrogenic cells [89•]. Following transplantation of induced chondrogenic cells into the subcutaneous spaces of nude mice, stable homogenous hyaline-like cartilage tissue formation was observed at 3 months, without evidence of tumors [89•]. Moreover, the induced chondrogenic cells formed cartilaginous tissue when implanted into articular cartilage defects in SCID rats and did not respond to osteogenic conditions. To date, only viral methods have been used to introduce the necessary reprogramming factors for conversion of fibroblasts to chondrocytes. Further studies are necessary to evaluate whether induced chondrogenic cells can be generated through the use of non-viral, integration-free methods and provide long-term in vivo hyaline cartilage repair.

There has been significant growth in the field of articular cartilage regeneration through the use of animal models of cartilage defects. However, some critical limitations remain. A recent review article by Sakata et al. provides an excellent summary of animal models of articular cartilage defects and some key perspectives regarding the challenges in interpreting these models [92]. Currently, there is no standardized outcome assessment protocol for true articular cartilage regeneration. A brief survey of the literature demonstrates that there are a plurality of morphometric stains, immunohistochemical approaches, gene expression analysis targets, and histologic grading scales utilized in the assessment of cartilage regeneration [93-96]. Unfortunately, there is significant variation in the specific outcome measures that are assessed for any particular study. With such heterogeneity, direct comparisons of the quality of repair tissue amongst multiple studies are highly challenging. Clearly, in some scenarios, a unique assessment may be needed to test a certain hypothesis. However, there is a critical need in the field for a basic consensus protocol, indicating which specific tests are absolutely required, for the accurate assessment of cartilage regeneration and the direct comparison with previous work.

\section{Conclusions and Future Perspectives}

Continued research on the instructive signaling cues and epigenetic mechanisms that control chondrogenic lineage commitment and articular chondrocyte fate specification will prove essential for advancing our fundamental knowledge of cartilage development as well as regenerative applications of the future. Many different strategies employing varying combinations of growth factors, progenitor-like populations, and culture conditions are currently being investigated for the formation of hyaline cartilage from human embryonic and induced pluripotent stem cells. The "optimal" strategy for producing articular-like chondrocytes that maintain functional and genomic integrity in vivo and contribute to articular cartilage defect repair remains elusive and will require more comprehensive studies in vitro and in vivo. Despite the significant progress made toward to the use of safer viral-free and nonintegrating reprogramming methods, concerns about the genome integrity of human iPSC-differentiated progeny and their safety still persist. Thus, there remains a critical need to apply more rigorous characterization of the differentiated cells as well as the removal of contaminating undifferentiated and undefined cells prior to in vivo transplantations. The idea that iPSCs may be used as an "off-the-shelf" product for cartilage repair will also require consideration of the time, effort, and costly resources to fully reprogram patient-derived cells, perform critical quality control analyses, and generate the desired cell type in high purity and sufficient quantity. Lineage reprogramming into cartilage-specific progenitor cells may provide a more efficient and reproducible approach and warrants further investigations.

Currently, there are no preclinical or clinical studies examining the potential for human iPSCs to repair cartilage. Indeed, several laboratories have begun to establish human iPSCs as platforms to model OA and to identify new targets for treating OA. Perhaps, the most imminent translational application of human iPSCs comes as a scalable source of patient- and disease-specific cells for small molecule and drug screening 
platforms to identify novel disease-modifying drugs [97]. Cartilage progenitors and articular chondrocytes derived from panels of patient-specific iPSCs may provide new opportunities for the discovery of molecules that stimulate chondrogenesis or drugs that prevent cartilage degeneration.

\section{Compliance with Ethical Standards}

Conflict of Interest Rosa M. Guzzo and Michael B. O'Sullivan declare that they have no conflict of interest.

Human and Animal Rights and Informed Consent This article does not contain any studies with human or animal subjects performed by any of the authors.

\section{References}

Papers of particular interest, published recently, have been highlighted as:

- Of importance

•- Of major importance

1. Hjelle K, Solheim E, Strand T, Muri R, Brittberg M. Articular cartilage defects in 1,000 knee arthroscopies. Arthroscopy. 2002;7:730-4

2. Widuchowski W, Widuchowski J, Trzaska T. Articular cartilage defects: study of 25,124 knee arthroscopies. Knee. 2007;14:17782 .

3. Safran MR, Seiber K. The evidence for surgical repair of articular cartilage in the knee. J Am Acad Orthop Surg. 2010;18:259-66.

4. Anderson AF, Dome DC, Gautam S, Awh MH, Rennirt GW. Correlation of anthropometric measurements, strength, anterior cruciate ligament size, and intercondylar notch characteristics to sex differences in anterior cruciate ligament tear rates. Am J Sports Med. 2001;29:58-66.

5. Jones CA, Pohar S. Health-related quality of life after total joint arthroplasty: a scoping review. Clin Geriatr Med. 2012;28:395429.

6. Aggarwal VK, Goyal N, Deirmengian G, Rangavajulla A, Parvizi J, Austin MS. Revision total knee arthroplasty in the young patient: is there trouble on the horizon? J Bone Joint Surg. 2014;96:536-42.

7. Maradit Kremers H, Larson DR, Crowson CS, Kremers WK, Washington RE, Steiner CA, et al. Prevalence of total hip and knee replacement in the United States. J Bone Joint Surg Am. 2015;97: 1386-97.

8. Brittberg $\mathrm{M}$, Lindahl A, Nilsson A, Ohlsson C, Isaksson $\mathrm{O}$, Peterson L. Treatment of deep cartilage defects in the knee with autologous chondrocyte transplantation. N Engl J Med. 1994;331: 889-95.

9. Peterson L, Vasiliadis HS, Brittberg M, Lindahl A. Autologous chondrocyte implantation: a long-term follow-up. Am J Sports Med. 2010;38:1117-24.

10. Brittberg M. Autologous chondrocyte implantation - technique and long-term follow-up. Injury. 2008;39 Suppl 1:S40-9.

11. Murphy MB, Moncivais K, Caplan AI. Mesenchymal stem cells: environmentally responsive therapeutics for regenerative medicine. Exp Mol Med. 2013;45:e54.
12. Pelttari K, Winter A, Steck E, Goetzke K, Hennig T, Ochs BG, et al. Premature induction of hypertrophy during in vitro chondrogenesis of human mesenchymal stem cells correlates with calcification and vascular invasion after ectopic transplantation in SCID mice. Arthritis Rheum. 2006;54:3254-66.

13. Reinisch A, Etchart N, Thomas D, Hofmann NA, Fruehwirth M, Sinha S, et al. Epigenetic and in vivo comparison of diverse MSC sources reveals an endochondral signature for human hematopoietic niche formation. Blood. 2015;125:249-60.

14. Vinardell T, Sheehy EJ, Buckley CT, Kelly DJ. A comparison of the functionality and in vivo phenotypic stability of cartilaginous tissues engineered from different stem cell sources. Tissue Eng Part A. 2012;18:1161-70.

15. Dickhut A, Pelttari K, Janicki P, Wagner W, Eckstein V, Egermann $\mathrm{M}$, et al. Calcification or dedifferentiation: requirement to lock mesenchymal stem cells in a desired differentiation stage. J Cell Physiol. 2009;219:219-26.

16. Zimmermann P, Boeuf S, Dickhut A, Boehmer S, Olek S, Richter W. Correlation of COL10A1 induction during chondrogenesis of mesenchymal stem cells with demethylation of two $\mathrm{CpG}$ sites in the COL10A1 promoter. Arthritis Rheum. 2008;58:2743-53.

17. Bara JJ, Richards RG, Alini M, Stoddart MJ. Concise review: bone marrow-derived mesenchymal stem cells change phenotype following in vitro culture: implications for basic research and the clinic. Stem Cells. 2014;32:1713-23.

18. Stolzing A, Jones E, McGonagle D, Scutt A. Age-related changes in human bone marrow-derived mesenchymal stem cells: consequences for cell therapies. Mech Ageing Dev. 2008;129:163-73.

19. Williams R, Khan IM, Richardson K, Nelson L, McCarthy HE, Analbelsi $\mathrm{T}$, et al. Identification and clonal characterisation of a progenitor cell sub-population in normal human articular cartilage. PLoS One. 2010;5:e13246.

20. Jiang Y, Tuan RS. Origin and function of cartilage stem/progenitor cells in osteoarthritis. Nat Rev Rheumatol. 2015;1:206-12.

21. Fickert S, Fiedler J, Brenner RE. Identification of subpopulations with characteristics of mesenchymal progenitor cells from human osteoarthritic cartilage using triple staining for cell surface markers. Arthritis Res Ther. 2004;6:R422-32.

22. Koelling S, Kruegel J, Irmer M, Path JR, Sadowski B, Miro X, et al. Migratory chondrogenic progenitor cells from repair tissue during the later stages of human osteoarthritis. Cell Stem Cell. 2009;4: 324-35.

23. Seol D, McCabe DJ, Choe H, Zheng H, Yu Y, Jang K, et al. Chondrogenic progenitor cells respond to cartilage injury. Arthritis Rheum. 2012;64:3626-37.

24. Lotz MK, Otsuki S, Grogan SP, Sah R, Terkeltaub R, D'Lima D. Cartilage cell clusters. Arthritis Rheum. 2010;62:2206-18.

25. Jiang Y, Hu C, Yu S, Yan J, Peng H, Ouyang HW, et al. Cartilage stem/progenitor cells are activated in osteoarthritis via interleukin1beta/nerve growth factor signaling. Arthritis Res Ther. 2015;17: 327.

26. Candela ME, Yasuhara R, Iwamoto M, Enomoto-Iwamoto M. Resident mesenchymal progenitors of articular cartilage. Matrix Biol. 2014;39:44-9.

27. Takahashi K, Tanabe K, Ohnuki M, Narita M, Ichisaka T, Tomoda $\mathrm{K}$, et al. Induction of pluripotent stem cells from adult human fibroblasts by defined factors. Cell. 2007;13:861-72.

28. Yu J, Vodyanik MA, Smuga-Otto K, Antosiewicz-Bourget J, Frane $\mathrm{JL}$, Tian S, et al. Induced pluripotent stem cell lines derived from human somatic cells. Science. 2007;318:1917-20.

29. Park IH, Zhao R, West JA, Yabuuchi A, Huo H, Ince TA, et al. Reprogramming of human somatic cells to pluripotency with defined factors. Nature. 2008;451:141-6.

30. Warren L, Manos PD, Ahfeldt T, Loh YH, Li H, Lau F, et al. Highly efficient reprogramming to pluripotency and directed differentiation 
of human cells with synthetic modified mRNA. Cell Stem Cell. 2010;7:618-30.

31. Yu J, Hu K, Smuga-Otto K, Tian S, Stewart R, Slukvin II, et al. Human induced pluripotent stem cells free of vector and transgene sequences. Science. 2009;324:797-801.

32. Okita K, Yamakawa T, Matsumura Y, Sato Y, Amano N, Watanabe A, et al. An efficient nonviral method to generate integration-free human-induced pluripotent stem cells from cord blood and peripheral blood cells. Stem Cells. 2013;31:458-66.

33. Yu J, Chau KF, Vodyanik MA, Jiang J, Jiang Y. Efficient feeder-free episomal reprogramming with small molecules. PLoS One. 2011;6: e17557.

34. Engle SJ, Vincent F. Small molecule screening in human induced pluripotent stem cell-derived terminal cell types. J Biol Chem. 2014;289:4562-70.

35. Liang P, Lan F, Lee AS, Gong T, Sanchez-Freire V, Wang Y, et al. Drug screening using a library of human induced pluripotent stem cell-derived cardiomyocytes reveals disease-specific patterns of cardiotoxicity. Circulation. 2013;127:1677-91.

36. Willems E, Cabral-Teixeira J, Schade D, Cai W, Reeves P, Bushway PJ, et al. Small molecule-mediated TGF-beta type II receptor degradation promotes cardiomyogenesis in embryonic stem cells. Cell Stem Cell. 2012;11:242-52.

37. Oh SW, Lee JB, Kim B, Jeon S, Kim MK, Nam KH, et al. Peptidomimetic small-molecule compounds promoting cardiogenesis of stem cells. Arch Pharm Res. 2012;35:1979-88.

38. Kunisada Y, Tsubooka-Yamazoe N, Shoji M, Hosoya M. Small molecules induce efficient differentiation into insulin-producing cells from human induced pluripotent stem cells. Stem Cell Res. 2012;8:274-84.

39. Ben-David U, Gan QF, Golan-Lev T, Arora P, Yanuka O, Oren YS, et al. Selective elimination of human pluripotent stem cells by an oleate synthesis inhibitor discovered in a high-throughput screen. Cell Stem Cell. 2013;12:167-79.

40. Wu L, Bluguermann C, Kyupelyan L, Latour B, Gonzalez S, Shah $\mathrm{S}$, et al. Human developmental chondrogenesis as a basis for engineering chondrocytes from pluripotent stem cells. Stem Cell Reports. 2013;1:575-89. This study was the first to isolate and distinguish the earliest cartilage-committed prechondrocytes and definitive chondrocytes during human chondrogenesis. A developmental paradigm for the generation and enrichment of iPSC-derived chondrocytes was established.

41.• Craft AM, Rockel JS, Nartiss Y, Kandel RA, Alman BA, Keller GM. Generation of articular chondrocytes from human pluripotent stem cells. Nat Biotechnol. 2015;33:638-45. This study demonstrated specification of a population of interzone cells and subsequent articular chondrocytes arising from hPSC-derived mesenchymal progenitors in response to sustained TGF $\beta$ signaling. iPSC-derived articular chondrocytes were used to model the early events that are known to participate in progression of OA.

42. Oldershaw RA, Baxter MA, Lowe ET, Bates N, Grady LM, Soncin $F$, et al. Directed differentiation of human embryonic stem cells toward chondrocytes. Nat Biotechnol. 2010;28:1187-94. The study reports the development of a chemically defined, 3-stage directed differentiation protocol that mimics the embryonic processes in chondrocyte lineage specification

43. Koyama N, Miura M, Nakao K, Kondo E, Fujii T, Taura D, et al. Human induced pluripotent stem cells differentiated into chondrogenic lineage via generation of mesenchymal progenitor cells. Stem Cells Dev. 2013;22:102-13.

44. Guzzo RM, Gibson J, Xu RH, Lee FY, Drissi H. Efficient differentiation of human iPSC-derived mesenchymal stem cells to chondroprogenitor cells. J Cell Biochem. 2013;114:480-90.

45. Umeda K, Zhao J, Simmons P, Stanley E, Elefanty A, Nakayama N. Human chondrogenic paraxial mesoderm, directed specification and prospective isolation from pluripotent stem cells. Sci Rep. 2012;2:455

46. Toh WS, Guo XM, Choo AB, Lu K, Lee EH, Cao T. Differentiation and enrichment of expandable chondrogenic cells from human embryonic stem cells in vitro. J Cell Mol Med. 2009;13:3570-90.

47. Nakagawa T, Lee SY, Reddi AH. Induction of chondrogenesis from human embryonic stem cells without embryoid body formation by bone morphogenetic protein 7 and transforming growth factor beta1. Arthritis Rheum. 2009;60:3686-92.

48. Bigdeli N, Karlsson C, Strehl R, Concaro S, Hyllner J, Lindahl A. Coculture of human embryonic stem cells and human articular chondrocytes results in significantly altered phenotype and improved chondrogenic differentiation. Stem Cells. 2009;27:181221.

49. Koay EJ, Athanasiou KA. Hypoxic chondrogenic differentiation of human embryonic stem cells enhances cartilage protein synthesis and biomechanical functionality. Osteoarthr Cartil. 2008;16:1450 6.

50. Koay EJ, Hoben GM, Athanasiou KA. Tissue engineering with chondrogenically differentiated human embryonic stem cells. Stem Cells. 2007;25:2183-90.

51. Goldring MB, Tsuchimochi K, Ijiri K. The control of chondrogenesis. J Cell Biochem. 2006;97:33-44.

52. Barna M, Niswander L. Visualization of cartilage formation: insight into cellular properties of skeletal progenitors and chondrodysplasia syndromes. Dev Cell. 2007;12:931-41.

53. Hatakeyama Y, Tuan RS, Shum L. Distinct functions of BMP4 and GDF5 in the regulation of chondrogenesis. J Cell Biochem. 2004;91:1204-17.

54. Koyama E, Shibukawa Y, Nagayama M, Sugito H, Young B, Yuasa $\mathrm{T}$, et al. A distinct cohort of progenitor cells participates in synovial joint and articular cartilage formation during mouse limb skeletogenesis. Dev Biol. 2008;316:62-73.

55. Spagnoli A, O'Rear L, Chandler RL, Granero-Molto F, Mortlock DP, Gorska AE, et al. TGF-beta signaling is essential for joint morphogenesis. J Cell Biol. 2007;177:1105-17.

56. Evseenko D, Zhu Y, Schenke-Layland K, Kuo J, Latour B, Ge S, et al. Mapping the first stages of mesoderm commitment during differentiation of human embryonic stem cells. Proc Natl Acad Sci U S A. 2010;107:13742-7.

$57 . \bullet$ Cheng A, Kapacee Z, Peng J, Lu S, Lucas RJ, Hardingham TE, et al. Cartilage repair using human embryonic stem cell-derived chondroprogenitors. Stem Cells Transl Med. 2014;3:1287-94. Optimization of a directed differentiation protocol in this study used feeder-free and serum-free hESC and iPSC culture systems to generate large numbers of chondrogenic cells. hESCderived chondrogenic cells promoted cartilage repair following implantation into focal osteochondral defects created in immunodeficient rats, without tumor formation.

58. Lee J, Taylor SE, Smeriglio P, Lai J, Maloney WJ, Yang F, et al. Early induction of a prechondrogenic population allows efficient generation of stable chondrocytes from human induced pluripotent stem cells. FASEB J. 2015;29:3399-410.

59.• Yamashita A, Morioka M, Yahara Y, Okada M, Kobayashi T, Kuriyama S, et al. Generation of scaffoldless hyaline cartilaginous tissue from human iPSCs. Stem Cell Reports. 2015;4:404-18. Transplantation of scaffoldless cartilaginous particles derived from hiPSCs into osteochondral defects in immunodeficient rats and immunosuppressed mini-pigs promoted neocartilage formation and integration into native cartilage. No tumor or ectopic tissue formation was observed following transplantation of hiPSC-derived cartilaginous particles in vivo.

60. Hwang NS, Varghese S, Lee HJ, Zhang Z, Ye Z, Bae J, et al. In vivo commitment and functional tissue regeneration using human embryonic stem cell-derived mesenchymal cells. Proc Natl Acad Sci U S A. 2008;105:20641-6. 
61. Nejadnik H, Diecke S, Lenkov OD, Chapelin F, Donig J, Tong X, et al. Improved approach for chondrogenic differentiation of human induced pluripotent stem cells. Stem Cell Rev. 2015;11:242-53.

62. Zheng YL, Sun YP, Zhang H, Liu WJ, Jiang R, Li WY, et al. Mesenchymal stem cells obtained from synovial fluid mesenchymal stem cell-derived induced pluripotent stem cells on a Matrigel coating exhibited enhanced proliferation and differentiation potential. PLoS One. 2015;10:e144226.

63. Lian Q, Lye E, Suan Yeo K, Khia Way Tan E, Salto-Tellez M, Liu TM, et al. Derivation of clinically compliant MSCs from CD105+, CD24- differentiated human ESCs. Stem Cells. 2007;25:425-36.

64. Ko JY, Kim KI, Park S, Im GI. In vitro chondrogenesis and in vivo repair of osteochondral defect with human induced pluripotent stem cells. Biomaterials. 2014;35:3571-81.

65. Liu Y, Goldberg AJ, Dennis JE, Gronowicz GA, Kuhn LT. One-step derivation of mesenchymal stem cell (MSC)-like cells from human pluripotent stem cells on a fibrillar collagen coating. PLoS One. 2012;7:e33225.

66. Wang X, Kimbrel EA, Ijichi K, Paul D, Lazorchak AS, Chu J, et al. Human ESC-derived MSCs outperform bone marrow MSCs in the treatment of an EAE model of multiple sclerosis. Stem Cell Reports. 2014;3:1-16.

67. Drissi H, Gibson JD, Guzzo RM, Xu RH. Derivation and chondrogenic commitment of human embryonic stem cellderived mesenchymal progenitors. Methods Mol Biol. 2015; 1340:65-78.

68. Chen YS, Pelekanos RA, Ellis RL, Horne R, Wolvetang EJ, Fisk NM. Small molecule mesengenic induction of human induced pluripotent stem cells to generate mesenchymal stem/stromal cells. Stem Cells Transl Med. 2012;1:83-95.

69. Barberi T, Willis LM, Socci ND, Studer L. Derivation of multipotent mesenchymal precursors from human embryonic stem cells. PLoS Med. 2005;2:e161.

70. Toh WS, Lee EH, Richards M, Cao T. In vitro derivation of chondrogenic cells from human embryonic stem cells. Methods Mol Biol. 2010;584:317-31.

71. Guzzo RM, Drissi H. Differentiation of human induced pluripotent stem cells to chondrocytes. Methods Mol Biol. 2015;1340:79-95.

72. Gong G, Ferrari D, Dealy CN, Kosher RA. Direct and progressive differentiation of human embryonic stem cells into the chondrogenic lineage. J Cell Physiol. 2010;224:664-71.

73. Diederichs S, Tuan RS. Functional comparison of human-induced pluripotent stem cell-derived mesenchymal cells and bone marrowderived mesenchymal stromal cells from the same donor. Stem Cells Dev. 2014;23:1594-610.

74. Dominici M, Le Blanc K, Mueller I, Slaper-Cortenbach I, Marini F, Krause D, et al. Minimal criteria for defining multipotent mesenchymal stromal cells. The International Society for Cellular Therapy position statement. Cytotherapy. 2006;8:315-7.

75. Goldberg AD, Allis CD, Bernstein E. Epigenetics: a landscape takes shape. Cell. 2007;128:635-8.

76. Kouzarides T. Chromatin modifications and their function. Cell. 2007;128:693-705.

77. Herlofsen SR, Bryne JC, Hoiby T, Wang L, Issner R, Zhang X, et al. Genome-wide map of quantified epigenetic changes during in vitro chondrogenic differentiation of primary human mesenchymal stem cells. BMC Genomics. 2013;14:105. Whole genome chromatin immunoprecipitation and deep sequencing for quantification of histone modifications, DNA methylation analyses, and mRNA microarrays were used to correlate changes in gene expression with epigenomic modifications during chondrogenic differentiation of human bone marrow-derived MSCs.

78. Li J, Ohliger J, Pei M. Significance of epigenetic landscape in cartilage regeneration from the cartilage development and pathology perspective. Stem Cells Dev. 2014;23:1178-94.
79. El-Serafi AT, Oreffo RO, Roach HI. Epigenetic modifiers influence lineage commitment of human bone marrow stromal cells: differential effects of 5-aza-deoxycytidine and trichostatin A. Differentiation. 2011;81:35-41.

80. Lee HL, Yu B, Deng P, Wang CY, Hong C. TGF-beta-induced KDM4B promotes chondrogenic differentiation of human MSCs. Stem cells. 2015 Oct 20 [Epub ahead of print]

81. Zhang F, Xu L, Xu Q, Li D, Yang Y, Karsenty G, et al. JMJD3 promotes chondrocyte proliferation and hypertrophy during endochondral bone formation in mice. J Mol Cell Biol. 2015;7:23-34.

82. Im GI, Shin KJ. Epigenetic approaches to regeneration of bone and cartilage from stem cells. Expert Opin Biol Ther. 2015;15:181-93.

83. Hata K, Takashima R, Amano K, Ono K, Nakanishi M, Yoshida M, et al. Arid $5 \mathrm{~b}$ facilitates chondrogenesis by recruiting the histone demethylase Phf2 to Sox9-regulated genes. Nat Commun. 2013;4: 2850.

84. Liu J, Nie H, Xu Z, Niu X, Guo S, Yin J, et al. The effect of 3D nanofibrous scaffolds on the chondrogenesis of induced pluripotent stem cells and their application in restoration of cartilage defects. PLoS One. 2014;9:e111566.

85. Cheng A, Hardingham TE, Kimber SJ. Generating cartilage repair from pluripotent stem cells. Tissue Eng B Rev. 2014;20:257-66.

86. Diekman BO, Christoforou N, Willard VP, Sun H, Sanchez-Adams J, Leong KW, et al. Cartilage tissue engineering using differentiated and purified induced pluripotent stem cells. Proc Natl Acad Sci U S A. 2012;109:19172-7.

87. Saito T, Yano F, Mori D, Kawata M, Hoshi K, Takato T, et al. Hyaline cartilage formation and tumorigenesis of implanted tissues derived from human induced pluripotent stem cells. Biomed Res. 2015;36:179-86. This study reported teratoma formation following transplantation of differentiated iPSCs into the articular cartilage of NOD/SCID mouse knee joint. Results of this study highlight the need to apply rigorous characterization of the differentiated cells as well as the removal of contaminating undifferentiated and undefined cells prior to in vivo transplantation.

88. Olee T, Grogan SP, Lotz MK, Colwell Jr CW, D’Lima DD, Snyder EY. Repair of cartilage defects in arthritic tissue with differentiated human embryonic stem cells. Tissue Eng Part A. 2014;20:683-92.

89. Outani H, Okada M, Yamashita A, Nakagawa K, Yoshikawa H, Tsumaki N. Direct induction of chondrogenic cells from human dermal fibroblast culture by defined factors. PLoS One. 2013;8:e77365. This study identified the reprogramming and chondrogenic factors sufficient to induce the formation of chondrogenic cells from human dermal fibroblasts, without the generation of pluripotent stem cells.

90. Bar-Nur O, Verheul C, Sommer AG, Brumbaugh J, Schwarz BA, Lipchina I, et al. Lineage conversion induced by pluripotency factors involves transient passage through an iPSC stage. Nat Biotechnol. 2015;33:761-8.

91. Maza I, Caspi I, Zviran A, Chomsky E, Rais Y, Viukov S, et al. Transient acquisition of pluripotency during somatic cell transdifferentiation with iPSC reprogramming factors. Nat Biotechnol. 2015;33:769-74.

92. Sakata R, Iwakura T, Reddi AH. Regeneration of articular cartilage surface: morphogens, cells, and extracellular matrix scaffolds. Tissue Eng B Rev. 2015;21:461-73.

93. Wakitani S, Goto T, Pineda SJ, Young RG, Mansour JM, Caplan AI, et al. Mesenchymal cell-based repair of large, full-thickness defects of articular cartilage. J Bone Joint Surg Am. 1994;76:57992.

94. O'Driscoll SW, Keeley FW, Salter RB. The chondrogenic potential of free autogenous periosteal grafts for biological resurfacing of major full-thickness defects in joint surfaces under the influence of continuous passive motion. An experimental investigation in the rabbit. J Bone Joint Surg Am. 1986;68:1017-35.

95. Mainil-Varlet P, Aigner T, Brittberg M, Bullough P, Hollander A, Hunziker E, et al. Histological assessment of cartilage repair: a 
report by the Histology Endpoint Committee of the International Cartilage Repair Society (ICRS). J Bone Joint Surg Am. 2003;85-A Suppl 2:45-57.

96. Mainil-Varlet P, Van Damme B, Nesic D, Knutsen G, Kandel R, Roberts S. A new histology scoring system for the assessment of the quality of human cartilage repair: ICRS II. Am J Sports Med. 2010;38:880-90.
97. Yang SL, Harnish E, Leeuw T, Dietz U, Batchelder E, Wright PS, et al. Compound screening platform using human induced pluripotent stem cells to identify small molecules that promote chondrogenesis. Protein Cell. 2012;3:934-42. 